\title{
Osteosynthese mit Fixateur externe
}

\author{
Hans-Jörg Oestern
}

\section{Zusammenfassung}

Die Fixateur-externe-Osteosynthese der distalen Radiusfraktur ist indiziert bei offenen Frakturen, A 3-Frakturen mit ausgedehnter dorsaler Trümmerzone, intraartikulären C 2- und C 3Frakturen sowie infizierten Frakturen z.B. nach vorausgegangener K-Drahtosteosynthese. Das biomechanische Prinzip beruht auf der Ligamentotaxis und ist an einen intakten dorsalen Bandapparat gebunden. Neben dem transartikulären Fixateur finden unterschiedliche Modelle des Bewegungsfixateurs ihre Anwendung ebenso wie die Anordnung eines Fixateurs im proximalen und distalen Fragment unter Ermöglichung der Beweglichkeit im Handgelenk. Die Ruhigstellungsdauer sollte 3-4 Wochen nicht überschreiten. Deshalb ist die zusätzliche Einbringung von Kirschner-Drähten zu empfehlen. Auto-, homologe Spongiosaplastik sowie Knochenersatzstoffe sind zur Auffüllung des dorsalen Defektbereiches in gleicher Weise geeignet. Als Komplikationen können Infekte im Bereich der Schanz'schen Schrauben auftreten, ebenso Irritationen des N. radialis superficialis. Eine zu lange Distraktion führt $\mathrm{zu}$ einer sympathischen Reflexdystrophie. Unter bestimmten Indikationen sollte ein Verfahrenswechsel möglichst frühzeitig nach Weichteilsituation erfolgen.

\section{Einleitung}

Trotz der Einführung der winkelstabilen Platte stellt der Fixateur externe auch mit einiger Evidenz [13] nach wie vor ein geeignetes Verfahren zur Behandlung stark dislozierter Frakturen sowie von Frakturen mit erheblicher Beteiligung der Gelenkfläche dar. Das Verfahren ist nicht neu und wurde bereits 1929 von Ombredanne [25] beschrieben und von Hoffmann [16] für die transartikuläre Montage mit einem Distraktionsmodul erweitert. Die transartikuläre Distraktion gelingt, da die kapsulären und ligamentären Strukturen gewöhnlich intakt sind. Das Prinzip ist bekannt unter dem Begriff Ligamentotaxis, eine Bezeichnung, die von Vidal [32] in die Literatur eingeführt wurde und auch von anderen Lokalisatio-

OP-JOURNAL 2003; 19: 28-32

(c) Georg Thieme Verlag Stuttgart . New York nen z. B. der Fixateur-interne-Instrumentation bei Wirbelfrakturen bekannt ist.

\section{Biomechanik}

Das Fixateur-externe-Prinzip basiert auf dem load transfer [6]. Ein Teil der Kraft oder die Gesamtheit der Kräfte, die normalerweise von den Ossa metacarpalia auf den Radius übertragen werden, wird durch den Fixateur überbrückt. Ein derartiger Lasttransfer hängt ab von der Steifigkeit des Fixateur externe.

Frykman und Mitarbeiter [12] haben die Fraktursteifigkeit verschiedener Fixateurmodelle untersucht. Der kleine AO-Fixateur hat dabei, ähnlich wie der Hoffmann-Fixateur, eine mittlere Steifigkeit, wobei natürlich die optimale Fraktursteifigkeit nicht bekannt ist.

In einer experimentellen Untersuchung von Bartosh und Saldana [4] wurde der Einfluss der Ligamentotaxis an einer experimentell erzeugten Frykman-Typ-VII-
Fraktur mit Beteiligung des radiocarpalen und radioulnaren Gelenkbereichs untersucht. Während der Ligamentotaxis kam es zu einem Zug an den palmaren Bändern und zu einer Distraktion des distalen Radiusfragmentes. Dieser Zug trat ein, bevor die dorsalen Bänder einen Effekt auf das distale Radiusfragment ausübten. Eine Beugung im Handgelenk während des Zuges vermehrte nur die akkordeonähnliche Wirkung der dorsalen Bänder und verhinderte eine effektive dorsale Ligamentotaxis. Die durchschnittliche Distraktion betrug $3 \mathrm{~mm}$. Die palmaren Bänder verhinderten nach Erreichen ihrer maximalen Bandlänge eine weitere Distraktion. Erst nach Durchtrennung der palmaren Bänder und weiterem Zug konnte der dorsale Bandapparat allein weiterwirken, so dass die Reposition möglich war.

Damit sind aus anatomischen und biomechanischen Gründen zusätzliche manuelle Maßnahmen zur Reposition notwendig.

Schuind et al. [29] führten die Reposition intraartikulärer Fragmente unter Distraktion auf die Verminderung des intraartikulären Druckes auf bis zu $-158 \mathrm{~mm}$ Hg zurück, Palmarflexion erhöht den Druck auf $91,8 \mathrm{~mm} \mathrm{Hg}$.

\section{Indikation für Fixateur externe}

- Offene Frakturen

- A 3 Frakturen mit ausgedehnter dorsaler Trümmerzone

- Intraartikuläre Frakturen (C 2 und C 3)

- Infizierte Frakturen (z.B. nach vorausgegangener K-Drahtfixierung)

\section{Osteosynthesetechnik}

Die Lagerung erfolgt auf einem Armtisch. Als günstig hat es sich erwiesen, zunächst eine manuelle Reposition durchzuführen und diese mit Hilfe von Kirschner-Drähten zu fixieren. Gelenktragende Fragmente können mit einem K-Draht von 
dorsal reponiert und anschließend durch K-Drähte fixiert werden. Die kleinen Schanz'schen Schrauben werden im 2. Metacarpale sowie im distalen Radius platziert, ungefähr $10-12 \mathrm{~cm}$ proximal der Spitze des Proc. styloideus radii $[23,24]$.

Seitz und andere (30] haben in anatomischen Untersuchungen nachgewiesen, dass hier die optimale Insertion liegt.

Unter Bildwandlerkontrolle werden die Einstichstellen markiert, anschließend erfolgten die Hautinzision und stumpfe Präparation mit der Schere, die Platzierung der Bohrhülse und der Schanz'schen Schrauben.

Das offene Vorgehen über eine kleine Hautinzision bietet mehr Sicherheit im Hinblick auf eine Nerven- oder Sehnenschädigung.

Die Schanz'schen Schrauben im Bereich des Os metacarpale werden im proximalen und im distalen Drittel eingebracht, um die sagittalen Fasern des Streckapparates über dem Metacarpophalangealgelenk zu schonen. Das Vorbohren im Metacarpale II erfolgt mit 2 mm, am Radiusschaft mit 2,7 mm. Der Pin-Durchmesser beträgt am Metacarpale $2-3 \mathrm{~mm}$ und am Radiusschaft 3-4 mm.

Um einen größeren Knochenkontakt im 2. Mittelhandknochen zu erreichen, können die Schanz'schen Schrauben auch in einem Winkel von etwa $30^{\circ}$ zur Achse des Os metacarpale eingebracht werden. Der Einbringwinkel zur Horizontalen beträgt ungefähr $45^{\circ}$, das Handgelenk sollte nach Anlegen der Fixateur-externe-Stangen in Neutralstellung oder bis $10^{\circ}$ Dorsalextension stehen.

Entsprechend biomechanischer Untersuchungen von Herzberg [15] kommt es durch die konvergierende Anordnung der Schanz'schen Schrauben zu einer Reduktion der axialen Druck- und Zugkräfte einerseits, andererseits jedoch zu einer Erhöhung der Biegekräfte.

Hutchinson [17] konnte in einer Analyse von 50 Fixateur-externe-Osteosynthesen beim Vergleich vorgebohrter gegenüber direkt gebohrter Schanz'scher Schrauben keine vermehrte Infektrate nachweisen, allerdings war die Temperatur bei direkter Bohrung an der Eintrittsstelle signifikant erhöht.
Der gelenküberbrückende Fixateur sollte nicht länger als 3 bis 4 Wochen belassen werden, da eine zu starke Distraktion die Gefahr der Gelenkeinsteifung und der sympathischen Reflexdystrophie mit Merk2 ssich bringt. Die K-Drähte gewährleisten dann anschließend genügend Stabilität bis zur Ausheilung (Abb.1).

Um die fehlende Beweglichkeit im Handgelenk durch einen transartikulären Fixateur zu vermeiden, existieren 2 Möglichkeiten:

1. Platzierung der Schanzschrauben in das distale Fragment z. B. bei A 3-Frakturen und

2. der Bewegungsfixateur, der 1987 von Clyburn [7] eingeführt und mittlerweile in verschiedenen Versionen vorliegt $[2,26]$.

Die Bewegungsachse des Handgelenkes projiziert sich auf den proximalen Anteil des Os capitatum. Bei Bewegungsstudien zeigt sich eine ellipsoide Bewegungsbahn mit Drehpunkt im Os capitatum. Diese Bewegungsbahn wurde in Form eines Bewegungsschlittens mit dem Bewegungsfixateur nach Asche [2] nachempfunden. Bei einem zugelassenen Bewegungsradius von $30^{\circ}$ hatten $92 \%$ bei einer Nachuntersuchung einen Ulnavorschub unter $2 \mathrm{~mm}$.

Jenkins et al. [18] konnten bei der Stabilisierung von distalen Radiusfrakturen in 94\% die Pins im distalen Fragment platzieren und damit eine Bewegung im Handgelenk bei guten Spätresultaten ermöglichen.

McQueen [22] konnte in vergleichenden Untersuchungen zwischen gelenküberbrückendem und nicht überbrückendem Fixateur deutlich bessere Ergebnisse in der Gruppe mit nicht überbrückendem Fixateur nachweisen und empfahl deshalb diese Anordnung für die Behandlung der distalen Radiusfrakturen.

\section{Zusatzmaßnahmen}

Duming [9] konnte experimentell nachweisen, dass eine alleinige Fixateur-externe-Ruhigstellung immer noch eine Bewegung der Fragmente ermöglichte. Wurden zusätzlich Kirschner-Drähte eingebracht, entsprach die Stabilität der der Plattenfixation. Ähnliche Ergebnisse zeigte Wolfe [35]. Die Kirschner-Draht-Fixierung führte zu einer deutlichen Verminderung der Bewegung des distalen Radiusfragmentes bei Beugung/Streckung, Radial- und Ulnarabduktion und Rotation.
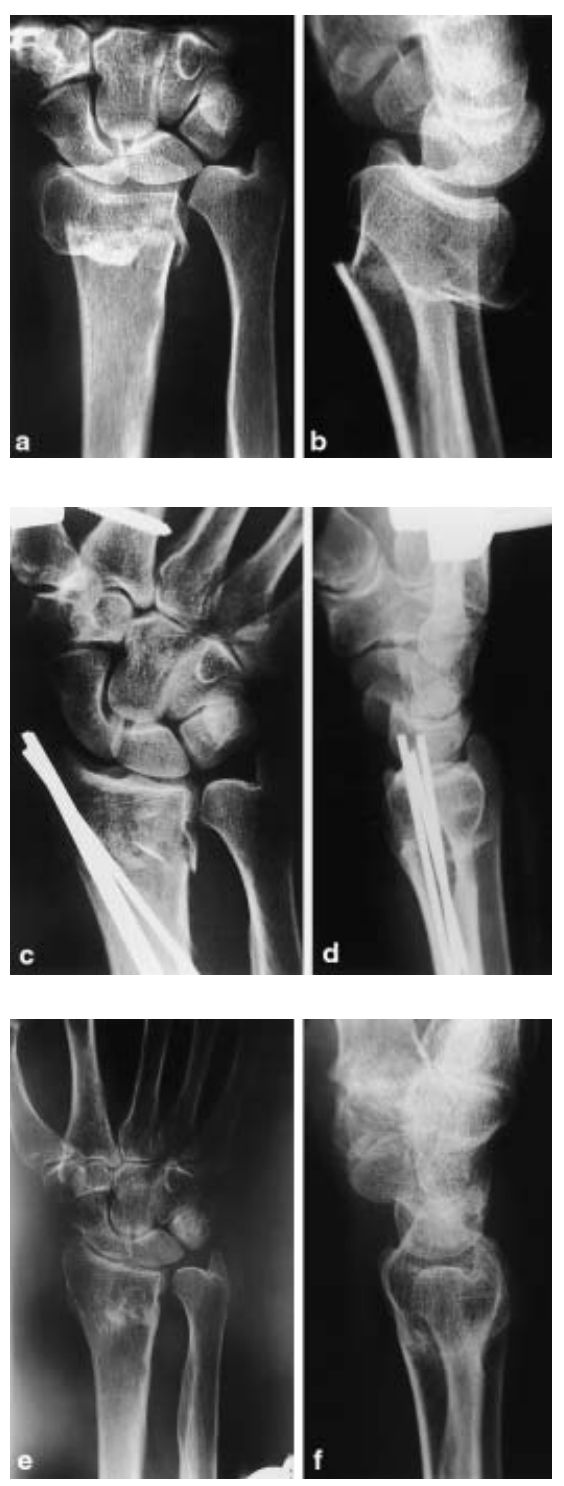

Abb.1 Extraartikuläre Fraktur des Radius, impaktiert mit axialer Verkürzung $(\mathbf{a}, \mathbf{b})$. Osteosynthese mit Kirschner-Drähten und Fixateur (c, d). Ausheilungsbilder 1 Jahr nach Unfall $(\mathbf{e}, \mathbf{f})$.

Bei ausgedehnten Spongiosadefekten mit Impression der Gelenkfläche dient der Fixateur vorerst der Längsdistraktion. Bei größeren Knochendefekten empfiehlt sich die primäre Durchführung einer Spongiosaplastik, um einem Korrekturverlust entgegenwirken zu können. Unter Distraktion kann auch über einen dorsalen Zugang das 2. und 3.Strecksehnenfach gespalten und dadurch das Gelenk inspiziert werden. Damit können die einzelnen gelenktragenden Fragmente aufgerichtet und die Defekthöhlen mit Spongiosa aufgefüllt werden (Abb.2). 

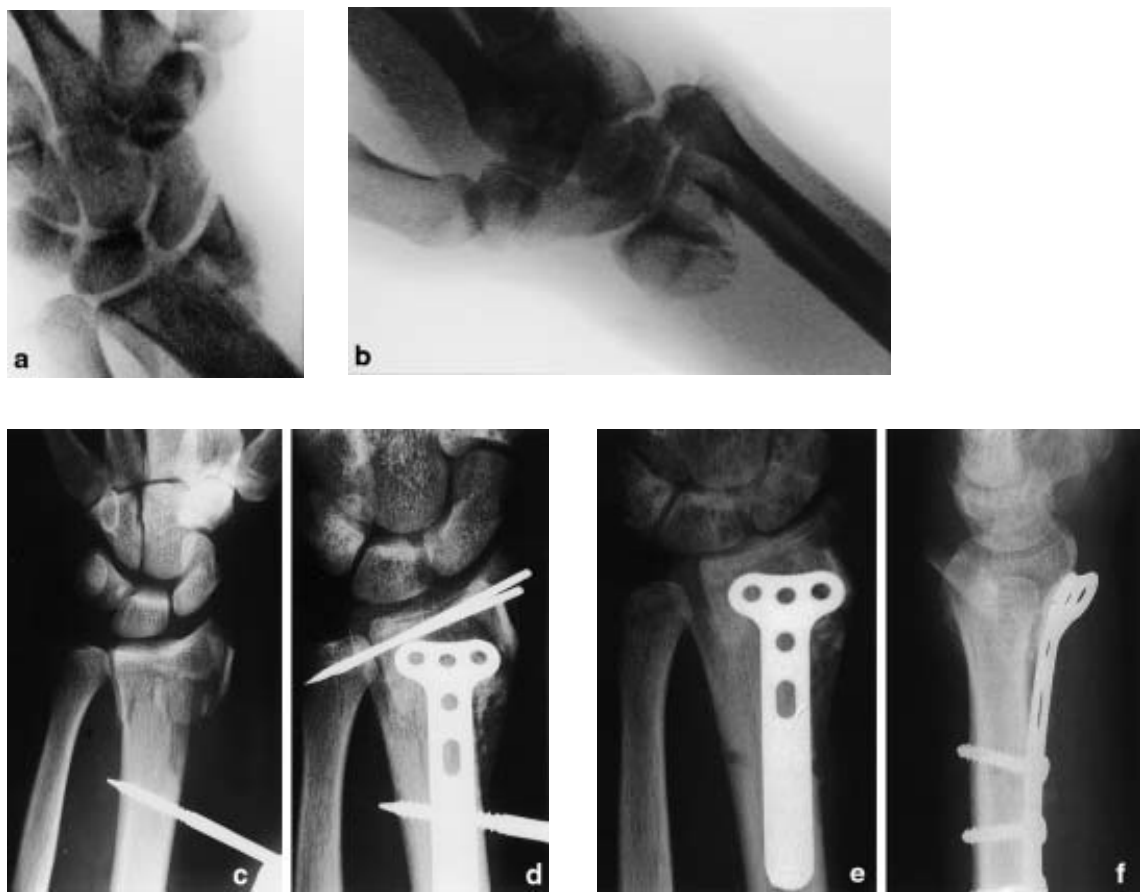

Abb. 2 Mehrfragmentäre artikuläre Fraktur mit volarer Dislokation (a, b). Primäre Anlage eines Fixateurs mit erheblicher Überdistraktion (c). Sekundäre Anlage einer volaren Platte und belassen des Fixateurs und Transfixierung des Radioulnargelenkes sowie Anlage einer Spongiosaplastik (d). Ausheilungsbilder mit noch liegender Platte (e, f). Funktion Pronation/Supination 90/0/90, Dorsalextension und Palmarflexion jeweils um $15^{\circ}$ zur Gegenseite eingeschränkt.

Zur Defektauffüllung verwendete Sakano [28] einen Hydroxyapatit-Spacer mit einem sehr guten Ergebnis bei 24 von 25 Patienten mit Frykman VI - VIII Frakturen. Ebenso empfahl Sennwald et al. [31] aufgrund einer Analyse von 70 Fällen Hydroxyapatit und Kalziumphosphat sowie autologe Spongiosa. Ineffektiv war in seiner Untersuchung Collagenmatrix. Herrera et al [14] berichteten über 8 gute, 6 mäßige und kein schlechtes Ergebnis unter Verwendung von homologen Transplantaten.

Dagegen konnte Widman [34] bei dreiwöchiger Fixateur-externe-Behandlung und anschließender Gipsruhigstellung, die eine Flexion im Handgelenk erlaubte, keine signifikanten Unterschiede in einer Gruppe mit oder ohne Knochentransplantation feststellen.

Durch die Ligamentotaxis kann nur in $50 \%$ eine zufriedenstellende Reposition von C 2- und C 3-Frakturen erreicht werden. Imprimierte, häufig auch rotierte Fragmente führen zu einer Inkongruenzarthrose. Eine Verkürzung des Radius mit Ulnavorschub von $1 \mathrm{~mm}$ vermindert die Kontaktfläche im distalen Radioulnargelenk um 23\%. Bei einem Ulnavorschub von $2 \mathrm{~mm}$ beträgt die Verringerung der Kontaktfläche sogar $42 \%$ [3].

\section{Ergebnisse}

Franck et al. [11] untersuchten in einer vergleichenden Studie je 20 Patienten, die mit einem nicht überbrückenden Fixateur externe oder mit Kirschner-Drähten und Gipsruhigstellung behandelt wurden. Obwohl die Ergebnisse nach 6 Monaten nur geringe Unterschiede aufwiesen, zeigten die Frühresultate eine bessere Beweglichkeit bei der mit Fixateur externe behandelten Gruppe.

Brug et al. [5] untersuchten 62 A- und CFrakturen, in 32 Fällen wurde zusätzlich Endobon appliziert. Eine Sudeck'sche Dystrophie wurde in 1,1\% beobachtet.

Cooney [8] verwendete den transartikulären Fixateur externe bei 60 Patienten mit einem mittleren Lebensalter von 63 Jahren. 88\% der Frakturen waren intraartikulär. Das Endergebnis war sehr gut oder gut in $87 \%$ und ausreichend in $13 \%$ der Fälle. Eine systematische Spongiosaplastik wurde nicht durchgeführt.

Leung u. Mitarb. [20] verwendeten bei jüngeren Patienten und intraartikulären Frakturen über 3 Wochen den Fixateur externe und anschließend einen Brace. Alle Patienten erhielten eine Spongiosaplastik. Die Ergebnisse waren in 90,4\% sehr gut und gut, in 9,6\% der Fälle waren die Ergebnisse ausreichend.

\section{Komplikationen}

Als Komplikationen wurden in der Literatur Algodystrophie, sekundärer Korrekturverlust, Infekt und Frakturen in den Löchern der Schanz'schen Schrauben erwähnt.

Eine Infektion an den Eintrittsstellen ist möglich, kann jedoch durch entsprechend sorgfältige und regelmäßige PinPflege vermieden werden.

Ahlborg et al. [1] berichteten über 314 instabile Frakturen, die mit Fixateur externe behandelt wurden. Die Rate der Komplikationen belief sich auf 27\%, 21\% hatten Infekte im Bereich der Schanz'schen Schrauben.

Frauen über 75 Jahre erlitten am häufigsten (17\%) einen Infekt durch die Schanz'schen Schrauben. 4\% hatten eine Fraktur im Bereich der Schanz'schen Schrauben. Rikli et al. [27] berichten über eine Algodystrophierate von $6 \%$.

Eine wesentliche Komplikation ist die Irritation des Nervus radialis superficialis beim Einbringen der Schanz'schen Schrauben. Mit der Spreiztechnik lässt sich diese Komplikation jedoch vermeiden.

Kuner et al. [19] berichteten bei 76 Frakturen (71\% C-Frakturen) über 16\% Irritationen des N. radialis superficialis. Eine Lösung dieses Problems stellt Emami [10] vor. Er beobachtete durch eine streng dorsale und nicht dorsoradiale Platzierung der Schanzschraube im Radius bei 40 Patienten keine Verletzung des oberflächlichen Radialisastes.

Wird der Einbringwinkel von $45^{\circ}$ im Bereich des Metacarpale nicht eingehalten, kann eine Verletzung der Strecksehnen erfolgen.

\section{Verfahrenswechsel}

Ein weiteres Problem ist der sekundäre Korrekturverlust.

Wentzensen et al. [33] berichteten über einen sekundären Korrekturverlust von $3,7 \mathrm{~mm}$ bei 37 Patienten. Bei einem Verfahrenswechsel mit Spongiosaplastik betrug der Korrekturverlust durchschnittlich $1,1 \mathrm{~mm}$ bzw. $1,0 \mathrm{~mm}$ bei Fixateur und Spongiosaplastik. 

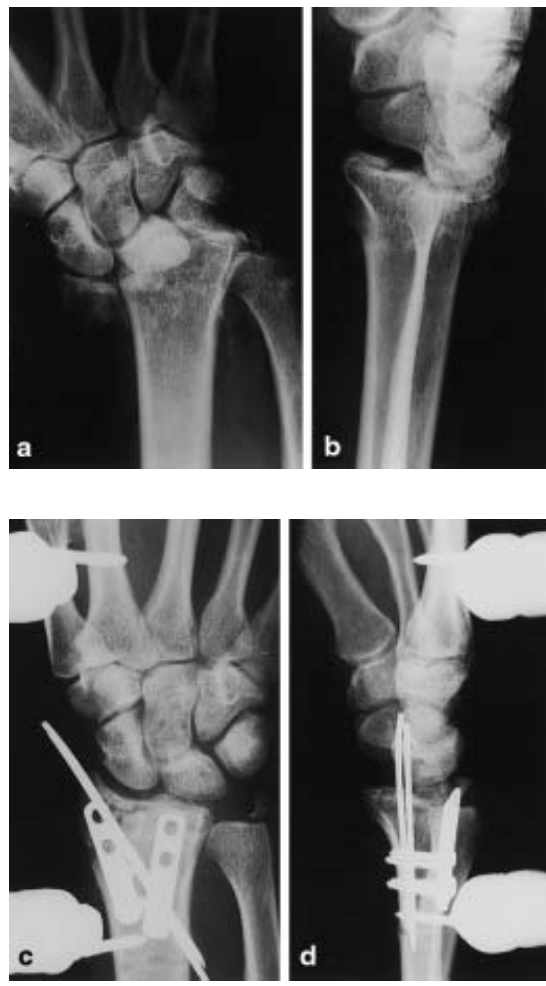

Abb.3 C 3-Fraktur (a, b) Anlage eines Fixateur externe. Die dorsalen Fragmente wurden mit 2 Platten und dorsal nur als Abstützung fixiert.

Die Indikation zum Verfahrenswechsel [21] besteht unter folgenden Bedingungen

nach Konsolidierung eines Weichteilschadens

- Nicht reponierbare Kantenfragmente (Abb. 3)

- Spongiosadefekt

nicht ausgeglichene Dorsalabkippung (Abb.4)

- Verkürzung des Radius

- Stufenbildung im Radiocarpal-/-ulnargelenk (Abb.5)

Die Behandlung der distalen Radiusfraktur mit dem Fixateur externe ist ein bewährtes Verfahren. Aufgrund unserer sehr guten Erfahrungen bei nun 107 distalen Radiusfrakturen mit der winkelstabilen Platte über einen volaren Zugang hat sich das Indikationsspektrum für den Fixateur externe in unserer Klinik auf die C 3-Frakturen und auf die seltenen offenen Frakturen Grad II und III reduziert.
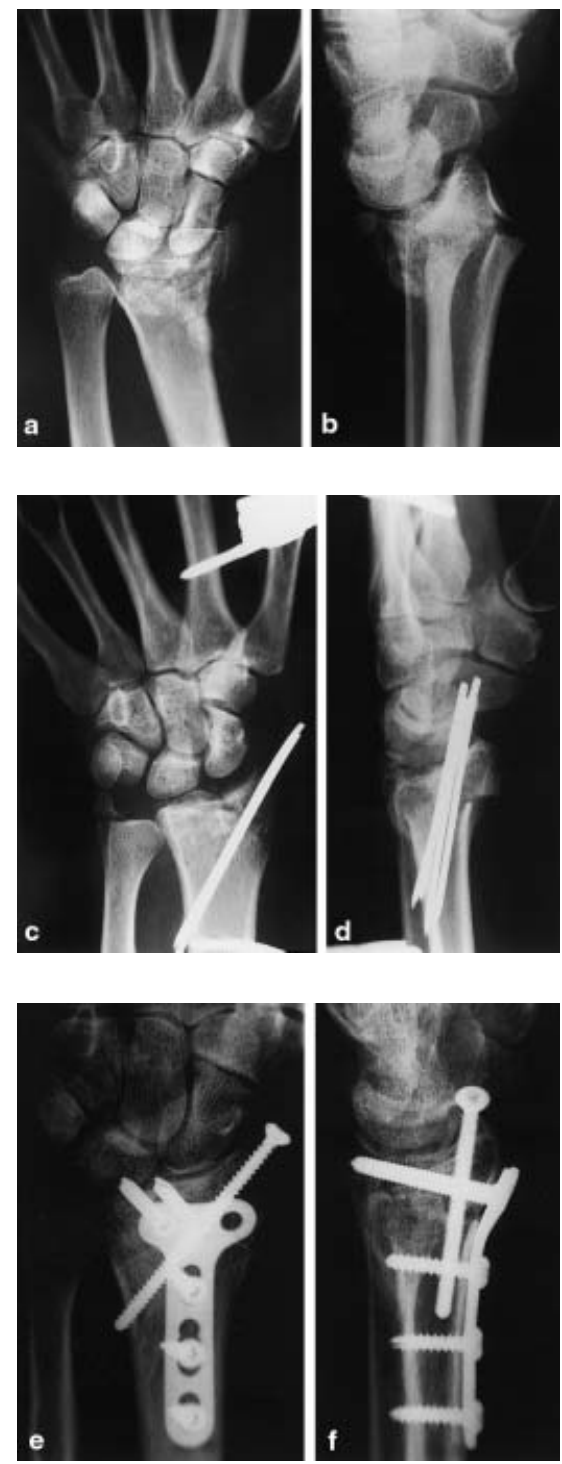

Abb. 4 C 3-Fraktur (a, b), primäre Osteosynthese mit Fixateur und Kirschner-Drähten (c, d). Verfahrenswechsel und Stabilisierung mit winkelstabiler Platte von volar und zusätzlicher Zugschraube zur Stabilisierung des Proc. styloideus radii $(\mathbf{e}, \mathbf{f})$.

\section{Literatur}

${ }^{1}$ Ahlborg HG, Josefsson PO. Pin-Tract complications in external fixation of fractures of the distal radius. Acta Orthop Scand 1999; 70: $116-118$

${ }^{2}$ Asche G. Die Behandlung von Speichenbrüchen mit einem neu entwickelten dynamischen Bewegungsfixateur. Z Chir 1995; 120: 952-958

${ }^{3}$ Bade H, Lobeck F. Gelenkflächenverhalten der Articulatio radioulnaris distalis bei fehlgestelltem distalem Radius. Unfallchirurgie 1991; 17: 213-217

${ }^{4}$ Bartosh RA, Saldana MJ. A cadaveric study to determine whether ligamentotaxis restores radiopalmar tili in intra-articular fractures of the distal radium. In: Fractures of the distal radius. Edited by Saffar P., Cooney III W.P.
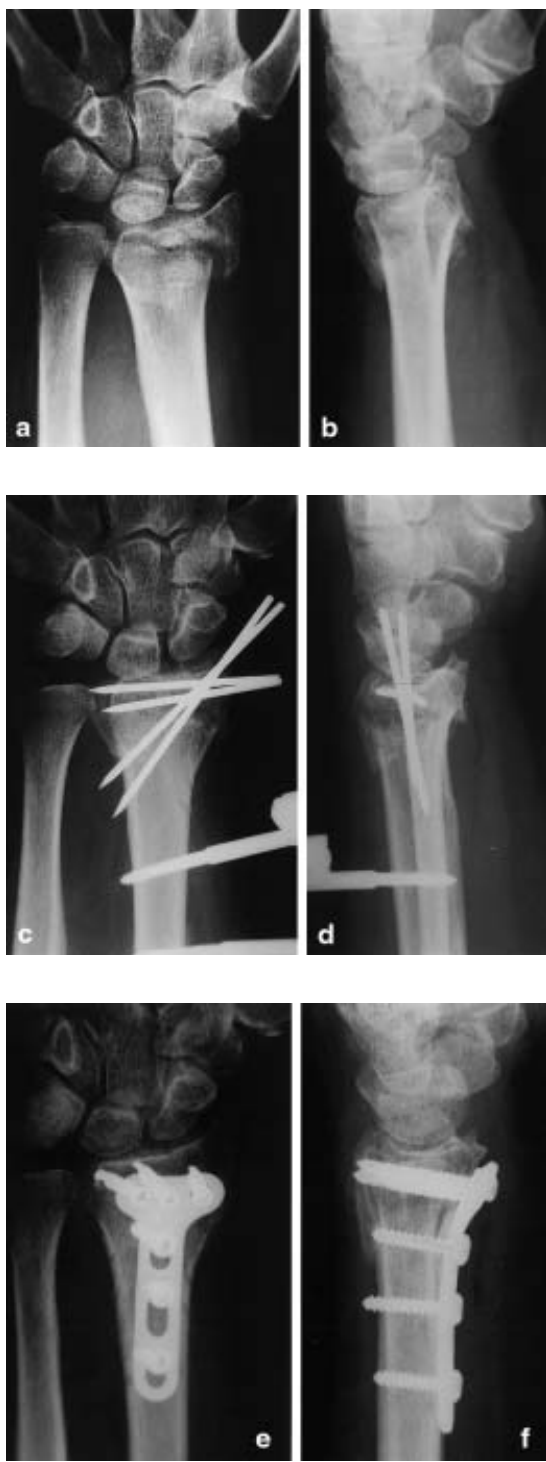

Abb.5 C 2-Fraktur (a, b), primäre Stabilisierung mit Kirschner-Drähten und Fixateur (c, d), Inkongruenz im Radioulnargelenk. Sekundäre Plattenosteosynthese von volar $(\mathbf{e}, \mathbf{f})$.

${ }^{5}$ Brug E, Joosten U, Pullen M. Fractures of the distal forearm. Which therapy is indicated when? Der Orthopäde 2000; 29: 318-326

${ }^{6}$ Chao EYS. Biomechanics of external fixation. In Lane J. Fracture Healing. New York: Churchill-Livingstone, 1987: 105-122

Clyburn TA. Dynamic external fixation for comminuted intraarticular fractures of the distal end of the radius. J Bone Jt Surg [A] 1987; 69: 248

${ }^{8}$ Cooney WP. External fixation of distal radial fractures. Clin Orthop 1993; 180: 44

${ }^{9}$ Duming CE, Lindsay CS, Bicknell RT, Patterson SD, Johnson JA, King GJ. Supplement pinning improves the stability of external fixation in distal radius fractures during simulated finger and forearm motion. J Hand Surg [Am] 1999; 24: 992-1000

${ }^{10}$ Emami A, Mjoberg B. A safer pin position for external fixation of distal radial fractures. Injury 2000; 31: 749-750 
${ }^{11}$ Franck WM, Dahlen C, Amlang M, Friese F, Zwipp H. Distal radius fracture - is nonbridging artivular external fixator a therapeutic alternative? A prospective randomized study. Unfallchirurg 2000; 103: 826-833

${ }^{12}$ Frykman K, Tooma GS. Comparion of 11 external fixators for treatment of unstable wrist fractures. J Hand Surg 1989; 14: 247254

${ }^{13}$ Handoll HH, Madhok R. Surgical interventions for treating distal radial fractures in adults. ACP J Club. 2002; 136: 112

${ }^{14}$ Herrera M, Chapman CB, Roh M, Strauch RJ, Rosenwasser MP. Treatment of unstable distal radius fractures with cancellou allograft and external fixation. J Hand Surg [Am] 1999; 24: 1269-78

${ }^{15}$ Herzberg W, Overbeck E, Herzberg R, Dekkelmann G. Vergleichende theoretische Untersuchung verschiedener Montagen des Fixateur externe zur Stabilisierung der distalen Radiusfraktur. Unfallchirurg 1990; 93 : 257-262

${ }^{16}$ Hoffmann R. Osteotaxis, osteosynthesis externe par finches et rotules. Acta Chir Scand 1954; 107: 72

${ }^{17}$ Hutchinson DT, Bachus KN, Higgenbotham T. External fixation of the distal radius: to predrill or not to predrill. J Hand Surg [m] 2000; 25: 1064-1068

${ }^{18}$ Jenkins NH, Jones DG, Johnson SR, MintowtCzyz WJ. External fixation of Colles' fractures. An anatomical study. JBJS [Br] 1987; 69: 207-211

${ }^{19}$ Kuner EH, Mellios K, Berwarth H. Treatment of complicated fracture of the distal radius with external fixatur. Follow-up-complications-outcomes. Unfallchirurg 2002; 105: 199-207
${ }^{20}$ Leung KS, Shen WY, Leung PC et al. Ligamentotaxis and bone grafting for comminuted fractures of the distal radius. J Bone Joint Surg [Br] 1989; 71: 838

${ }^{21}$ Matschke S, Heppert V, Winkler H, Wentzensen A. Der Verfahrenswechsel als Behandlungskonzept bei distalen Radiustrümmerfrakturen. In: Winker KH, Schmidt J: Der Stellenwert des externen Fixateurs bei der Behandlung der distalen Radiusfraktur. Thieme Verlag Stuttgart - New York 1999: 59-62

22 McQueen MM. Redisplace unstable fractures of the distal radius. A randomised, prospective study of bridging versus nonbridging external fixation. J Bone Joint Surg $[\mathrm{Br}]$ 1998; 80: 665-669

${ }^{23}$ Oestern HJ. Unterarmschaftfrakturen. In: Tscherne Unfallchirurgie Teil 1, Schmit-Neuerburg K.-P., Towfigh H, Letsch R. Springer Verlag Berlin - Heidelberg - New York 2001, 226-247

${ }^{24}$ Oestern HJ. Distale Radiusfrakturen. Operative Therapien. Chirurg 1999; 11: 1381-1394

${ }^{25}$ Ombredanne. L'osteosythese temporaire chez les enfants. Presse med 1929; 52

${ }^{26}$ Pennig D. Dynamic external fixation of distal radius fractures. Hand clinics 1993; 9: 587602

${ }^{27}$ Rikli D, Regazzoni P. Distal radium fractures. Schweiz Med Wochenschr 1999; 129: 776785

${ }^{28}$ Sakano H, Koshina T, Takeuchi R, Sakai N, Saito T. Treatment of the unstable distal radius fracture with external fixation and a hydroxyapatite spacer. J Hand Surg [Am] 2001; 26: 923-930

${ }^{29}$ Schuind FA, Cantraine FR, Fabeck L, Burny F. Radiocarpal articular pressures during the reduction of distal radius fractures. J Orthop Trauma 1997; 11: 295-299

${ }^{30}$ Seitz WH, Putman MD, Dick HM. Limited open surgical approach for external fixation of distal radius fractures. J Hand Surg 1990; 15 A: 288

${ }^{31}$ Sennwald G, Della Santa D. Utility of bone substitutes: study of 101 distal radius fractures. Chir Main 2001; 20: 454-457

${ }^{32}$ Vidal J, Buscayret C, Paran M et al. Ligamentotaxis. In: Mears, DC: External skeletal fixation. William \& Wilkins, Baltimore 1983: 493

${ }^{33}$ Wentzensen A, Matschke S. AO-Fixateur zur Behandlung distaler Speichenbrüche. In: Winker KH, Schmidt J: Der Stellenwert des externen Fixateurs bei der Behandlung der distalen Radiusfraktur. Thieme Verlag 1999: 5-7

${ }^{34}$ Widman J, Isacson J. Primary bone grafting does not improve the results in severely displaced distal radius fractures. Int Orthop 2002; 26: 20-22

${ }^{35}$ Wolfe SW, Austin G, Lorenze M, Swigart CR, Panjabi MM. A biomechanical comparison of different wrist external fixators with and without K-wire augmentation. J Hand Surg [Am] 1999; 24: 516-524

\section{Prof. Dr. med. Hans-Jörg Oestern} Chefarzt

Klinik für Unfall- und Wiederherstellungschirurgie

Allgemeines Krankenhaus Siemensplatz 4

D-29223 Celle 